\title{
AN EXACT GEOMETRIC MASS FORMULA
}

\author{
CHIA-FU YU
}

\begin{abstract}
We show an exact geometric mass formula for superspecial points in the reduction of any quaternionic Shimura variety modulo at a good prime $p$.
\end{abstract}

\section{INTRODUCTION}

Let $p$ be a rational prime number. Let $B$ be a totally indefinite quaternion algebra over a totally real field $F$ of degree $d$, together with a positive involution *. Assume that $p$ is unramified in $B$. Let $O_{B}$ be a maximal order stable under the involution $*$. Let $(V, \psi)$ be a non-degenerate $\mathbb{Q}$-valued skew-Hermitian (left) $B$-module with dimension $2 g$ over $\mathbb{Q}$. Put $m:=\frac{g}{2 d}$, a positive integer. A polarized abelian $O_{B}$-variety $\underline{A}=(A, \lambda, \iota)$ is a polarized abelian variety $(A, \lambda)$ together with a ring monomorphism $\iota: O_{B} \rightarrow \operatorname{End}(A)$ such that $\lambda \circ \iota\left(b^{*}\right)=\iota(b)^{t} \circ \lambda$ for all $b \in O_{B}$. Let $k$ be an algebraically closed field of characteristic $p$. An abelian variety over $k$ is said to be superspecial if it is isomorphic to a product of supersingular elliptic curves. Denote by $\Lambda_{g}^{B}$ the set of isomorphism classes of $g$-dimensional superspecial principally polarized abelian $O_{B}$-varieties over $k$. Define the mass of $\Lambda_{g}^{B}$ to be

$$
\operatorname{Mass}\left(\Lambda_{g}^{B}\right):=\sum_{\underline{A} \in \Lambda_{g}^{B}} \frac{1}{|\operatorname{Aut}(A, \lambda, \iota)|} .
$$

The mass Mass $\left(\Lambda_{g}^{B}\right)$ is studied in Ekedahl [1] (Ekedahl's result relies on an explicit volume computation in Hashimoto-Ibukiyama [4, Proposition 9, p. 568]) in the special case $B=M_{2}(\mathbb{Q})$. He proved

Theorem 1.1 (Ekedahl, Hashimoto-Ibukiyama). One has

$$
\operatorname{Mass}\left(\Lambda_{g}\right)=\frac{(-1)^{g(g+1) / 2}}{2^{g}} \prod_{i=1}^{g} \zeta(1-2 i) \cdot \prod_{i=1}^{g} p^{i}+(-1)^{i},
$$

where $\Lambda_{g}$ is the set of isomorphism classes of g-dimensional superspecial principally polarized abelian varieties over $k$ and $\zeta(s)$ is the Riemann zeta function.

Let $B_{p, \infty}$ be the quaternion algebra over $\mathbb{Q}$ ramified exactly at $\{p, \infty\}$. Let $B^{\prime}$ be the quaternion algebra over $F$ such that $\operatorname{inv}_{v}\left(B^{\prime}\right)=\operatorname{inv}_{v}\left(B_{p, \infty} \otimes_{\mathbb{Q}} B\right)$ for all $v$. Let $\Delta^{\prime}$ be the discriminant of $B^{\prime}$ over $F$.

In this paper we prove

Date: June 19, 2007. The research is partially supported by NSC 96-2115-M-001-001. 
Theorem 1.2. One has

$\operatorname{Mass}\left(\Lambda_{g}^{B}\right)=\frac{(-1)^{d m(m+1) / 2}}{2^{m d}} \prod_{i=1}^{m}\left\{\zeta_{F}(1-2 i) \prod_{v \mid \Delta^{\prime}} N(v)^{i}+(-1)^{i} \prod_{v \mid p, v \nmid \Delta^{\prime}} N(v)^{i}+1\right\}$, where $\zeta_{F}(s)$ is the Dedekind zeta function.

Let $N \geq 3$ be a prime-to- $p$ positive integer. Choose a primitive $n$-th root of unity $\zeta_{N} \in \overline{\mathbb{Q}} \subset \mathbb{C}$ and fix an embedding $\overline{\mathbb{Q}} \hookrightarrow \overline{\mathbb{Q}_{p}}$. Let $\mathcal{M}$ be the moduli space over $\overline{\mathbb{F}}_{p}$ of $g$-dimensional principally polarized abelian $O_{B}$-varieties with a symplectic $O_{B}$-linear level- $N$ structure w.r.t. $\zeta_{N}$. Let $L_{0}$ be a self-dual $O_{B}$-lattice of $V$ with respect to $\psi$. Let $G_{1}$ be the automorphism group scheme over $\mathbb{Z}$ associated to the pair $\left(L_{0}, \psi\right)$. As an immediate consequence of Theorem 1.2, we get

Theorem 1.3. The moduli space $\mathcal{M}$ has

$$
\left|G_{1}(\mathbb{Z} / N \mathbb{Z})\right| \frac{(-1)^{d m(m+1) / 2}}{2^{m d}} \prod_{i=1}^{m}\left\{\zeta_{F}(1-2 i) \prod_{v \mid \Delta^{\prime}} N(v)^{i}+(-1)^{i} \prod_{v \mid p, v \nmid \Delta^{\prime}} N(v)^{i}+1\right\}
$$

superspecial points.

We divide the proof of Theorem 1.2 into 4 parts; each part is treated in one section. The first part is to express the weighted sum in terms of an arithmetic mass; this is done in the author's recent work [8]. The second part is to compute the mass associated to a quaternion unitary group and a standard open compact subgroup; this is done by Shimura [7] (re-obtained by Gan and J.-K. Yu [3, 11.2, p. 522]) using the theory of Bruhat-Tits Buildings). The third part is to compare the derived arithmetic mass in Section 1 with "the" standard mass in Section 2. This reduces the problem to computing a local index at $p$. The last part uses Dieudonné theory to compute this local index. A crucial step is choosing a good basis for the superspecial Dieudonné module concerned; this makes the computation easier.

Notation. $\mathbb{H}$ denotes the Hamilton quaternion algebra over $\mathbb{R}$. $\mathbb{A}_{f}$ denotes the finite adele ring of $\mathbb{Q}$ and $\hat{\mathbb{Z}}=\prod_{p} \mathbb{Z}_{p}$. For a number field $F$ and a finite place $v$, denote by $O_{F}$ the ring of integers, $F_{v}$ the completion of $F$ at $v, e_{v}$ the ramification index for $F / \mathbb{Q}, \kappa_{v}$ the residue field, $f_{v}:=\left[\kappa_{v}: \mathbb{F}_{p}\right]$ and $q_{v}:=N(v)=\left|\kappa_{v}\right|$. For an $O_{F}$-module $A$, write $A_{v}$ for $A \otimes_{O_{F}} O_{F, v}$. For a scheme $X$ over Spec $A$ and an $A$ algebra $B$, write $X_{B}$ for $X \times_{\operatorname{Spec} A} \operatorname{Spec} B$. For a linear algebraic group $G$ over $\mathbb{Q}$ and an open compact subgroup $U$ of $G\left(\mathbb{A}_{f}\right)$, denote by $\operatorname{DS}(G, U)$ the double coset space $G(\mathbb{Q}) \backslash G\left(\mathbb{A}_{f}\right) / U$, and write $\operatorname{Mass}(G, U):=\sum_{i=1}^{h}\left|\Gamma_{i}\right|^{-1}$ if $G$ is $\mathbb{R}$-anisotropic, where $\Gamma_{i}:=G(\mathbb{Q}) \cap c_{i} U c_{i}^{-1}$ and $c_{1}, \ldots, c_{h}$ are complete representatives for $\operatorname{DS}(G, U)$. For a central simple algebra $B$ over $F$, write $\Delta(B / F)$ for the discriminant of $B$ over $F$. If $B$ a central division algebra over a non-archimedean local field $F_{v}$, denote by $O_{B}$ the maximal order of $B, \mathfrak{m}(B)$ the maximal ideal and $\kappa(B)$ the residue field. $\mathbb{Q}_{p^{n}}$ denotes the unramified extension of $\mathbb{Q}_{p}$ of degree $n$ and write $\mathbb{Z}_{p^{n}}:=O_{\mathbb{Q}_{p^{n}}}$.

\section{Simple MASS FORMULAS}

Let $B$ be a finite-dimensional semi-simple algebra over $\mathbb{Q}$ with a positive involution $*$, and $O_{B}$ be an order of $B$ stable under $*$. Let $k$ be any field. 
To any polarized abelian $O_{B}$-varieties $\underline{A}=(A, \lambda, \iota)$ over $k$, we associate a pair $\left(G_{x}, U_{x}\right)$, where $G_{x}$ is the group scheme over $\mathbb{Z}$ representing the functor

$$
R \mapsto\left\{h \in\left(\operatorname{End}_{O_{B}}\left(A_{k}\right) \otimes R\right)^{\times} \mid h^{\prime} h=1\right\},
$$

where $h \mapsto h^{\prime}$ is the Rasoti involution, and $U_{x}$ is the open compact subgroup $G_{x}(\hat{\mathbb{Z}})$. For any prime $\ell$, we write $\underline{A}(\ell)$ for the associated $\ell$-divisible group with additional structures $\left(A\left[\ell^{\infty}\right], \lambda_{\ell}, \iota_{\ell}\right)$, where $\lambda_{\ell}$ is the induced quasi-polarization from $A\left[\ell^{\infty}\right]$ to $A^{t}\left[\ell^{\infty}\right]=A\left[\ell^{\infty}\right]^{t}$ (the Serre dual), and $\iota_{\ell}: O_{B} \otimes \mathbb{Z}_{\ell} \rightarrow \operatorname{End}\left(A\left[\ell^{\infty}\right]\right)$ the induced ring monomorphism. For any two objects $\underline{A}_{1}$ and $\underline{A}_{2}$ over $k$, denote by Q-isom ${ }_{k}\left(\underline{A}_{1}, \underline{A}_{2}\right)$ the set of $O_{B}$-linear quasi-isogenies $\varphi: A_{1} \rightarrow A_{2}$ over $k$ such that $\varphi^{*} \lambda_{2}=\lambda_{1}$, and $\operatorname{Isom}_{k}\left(\underline{A}_{1}(\ell), \underline{A}_{2}(\ell)\right)$ the set of $O_{B} \otimes \mathbb{Z}_{\ell}$-linear isomorphisms $\varphi: A_{1}\left[\ell^{\infty}\right] \rightarrow A_{2}\left[\ell^{\infty}\right]$ over $k$ such that $\varphi^{*} \lambda_{2}=\lambda_{1}$.

Let $x:=\underline{A}_{0}=\left(A_{0}, \lambda_{0}, \iota_{0}\right)$ be a fixed polarized abelian $O_{B}$-variety over $k$. Denote by $\Lambda_{x}(k)$ the set of isomorphisms classes of polarized abelian $O_{B}$-varieties $\underline{A}$ over $k$ such that

$\left(I_{\ell}\right): \operatorname{Isom}_{k}\left(\underline{A}_{0}(\ell), \underline{A}(\ell)\right) \neq \emptyset$ for all primes $\ell$.

Let $\Lambda_{x}^{\prime}(k) \subset \Lambda_{x}(k)$ be the subset consisting of objects such that

(Q): $\mathrm{Q}-\operatorname{isom}_{k}\left(\underline{A}_{0}, \underline{A}\right) \neq \emptyset$.

Let $\operatorname{ker}^{1}\left(\mathbb{Q}, G_{x}\right)$ denote the kernel of the local-global map $H^{1}\left(\mathbb{Q}, G_{x}\right) \rightarrow \prod_{v} H^{1}\left(\mathbb{Q}_{v}, G_{x}\right)$.

Theorem 2.1. ([8, Theorem 2.3]) Suppose that $k$ is a field of finite type over its prime field.

(1) There is a natural bijection $\Lambda_{x}^{\prime}(k) \simeq \operatorname{DS}\left(G_{x}, U_{x}\right)$. Consequently, $\Lambda_{x}^{\prime}(k)$ is finite.

(2) One has $\operatorname{Mass}\left(\Lambda_{x}^{\prime}(k)\right)=\operatorname{Mass}\left(G_{x}, U_{x}\right)$.

Theorem 2.2. ([8, Theorem 4.6 and Remark 4.7]) Notation as above. If $k \supset \mathbb{F}_{p}$ is algebraically closed and $A_{0}$ is supersingular, then $\operatorname{Mass}\left(\Lambda_{x}^{\prime}(k)\right)=\operatorname{Mass}\left(G_{x}, U_{x}\right)$ and $\operatorname{Mass}\left(\Lambda_{x}(k)\right)=\left|\operatorname{ker}^{1}\left(\mathbb{Q}, G_{x}\right)\right| \cdot \operatorname{Mass}\left(G_{x}, U_{x}\right)$.

Remark 2.3. The statement of Theorem 2.2 is valid for basic abelian $O_{B}$-varieties in the sense of Kottwitz (see [6] for the definition). The present form is enough for our purpose.

\section{An exact mass formula of Shimura}

Let $D$ be a totally definite quaternion division algebra over a totally real field $F$ of degree $d$. Let (bar) $d \mapsto \bar{d}$ denote the canonical involution. Let $\left(V^{\prime}, \varphi\right)$ be a $D$-valued totally definite quaternion Hermitian $D$-module of rank $m$. Let $G^{\varphi}$ denote the unitary group attached to $\varphi$. This is a reductive group over $F$ and is regarded as a group over $\mathbb{Q}$ via the Weil restriction of scalars from $F$ to $\mathbb{Q}$. Choose a maximal order $O_{D}$ of $D$ stable under the canonical involution ${ }^{-}$. Let $L$ be an $O_{D}$-lattice in $V^{\prime}$ which is maximal among the lattices on which $\varphi$ takes its values in $O_{B}$. Let $U_{0}$ be the open compact subgroup of $G^{\varphi}\left(\mathbb{A}_{f}\right)$ which stabilizes the adelic lattice $L \otimes_{\mathbb{Z}} \hat{\mathbb{Z}}$.

The following is deduced from a mass formula of Shimura [7] (also see Gan J.-K. Yu [3, 11.2, p. 522]). This form is more applicable to prove Theorem 1.2. 
Theorem 3.1 (Shimura). One has

$$
\operatorname{Mass}\left(G^{\varphi}, U_{0}\right)=\frac{(-1)^{d m(m+1) / 2}}{2^{m d}} \prod_{i=1}^{m}\left\{\zeta_{F}(1-2 i) \prod_{v \mid \Delta(D / F)} N(v)^{i}+(-1)^{i}\right\} .
$$

Deduction. In [7, Introduction, p. 68] Shimura gives the explicit formula

$$
\operatorname{Mass}\left(G^{\varphi}, U_{0}\right)=\left|D_{F}\right|^{m^{2}} \prod_{i=1}^{m} D_{F}^{1 / 2}\left[(2 i-1) !(2 \pi)^{-2 i}\right]^{d} \zeta_{F}(2 i) \cdot \prod_{v \mid \Delta(D / F)} \prod_{i=1}^{m} N(v)^{i}+(-1)^{i},
$$

where $D_{F}$ is the discriminant of $F$ over $\mathbb{Q}$. Using the functional equation for $\zeta_{F}(s)$, we deduce (3.1) from (3.2).

\section{Global comparison}

Keep the notation as in Section 1 Fix a $g$-dimensional superspecial principally polarized abelian $O_{B}$-variety $x=\left(A_{0}, \lambda_{0}, \iota_{0}\right)$ over $k$. Define $\Lambda_{x}:=\Lambda_{x}(k)$ as in Section 2. Let $\left(G_{x}, U_{x}\right)$ be the pair associated to $x$.

Lemma 4.1. Any two self-dual $O_{B} \otimes \mathbb{Z}_{p}$-lattices of $\left(V_{\mathbb{Q}_{p}}, \psi\right)$ are isomorphic.

Proof. The proof is elementary and omitted.

Lemma 4.2. One has (1) $\Lambda_{x}=\Lambda_{g}^{B}$ (2) $\operatorname{ker}^{1}\left(\mathbb{Q}, G_{x}\right)=\{1\}$.

Proof. (1) The inclusion $\Lambda_{x} \subset \Lambda_{g}^{B}$ is clear. We show the other direction. Let $\underline{A} \in \Lambda_{g}^{B}$. It follows from Lemma 4.1 that the condition $\left(I_{\ell}\right)$ is satisfied for primes $\ell \neq p$. Let $M$ be the covariant Dieudonné module of $A$. One chooses an isomorphism $O_{B, p} \simeq M_{2}\left(O_{F, p}\right)$ so that $*:\left(a_{i j}\right) \mapsto\left(a_{i j}\right)^{t}$. Using the Morita equivalence, it suffices to show that any two superspecial principally quasi-polarized Dieudonné modules with compatible $O_{F, p}$-action are isomorphic. This follows from Theorem 5.1.

(2) Since $G_{x}$ is semi-simple and simply connected (as it is an inner form of $\left.\operatorname{Res}_{F / \mathbb{Q}} \operatorname{Sp}_{2 m, F}\right)$, the Hasse principle for $G_{x}$ holds.

4.1. We compute that

(i) $G_{x}(\mathbb{R})=\left\{h \in M_{m}(\mathbb{H})^{d} \mid \bar{h}^{t} h=1\right\}$,

(ii) for $\ell \neq p$, we have $G_{x}\left(\mathbb{Q}_{\ell}\right)=\prod_{v \mid \ell} G_{x, v}$ and $U_{x, \ell}=\prod_{v \mid \ell} U_{x, v}$, where

$$
\begin{aligned}
G_{x, v} & = \begin{cases}\operatorname{Sp}_{2 m}\left(F_{v}\right), & \text { if } v \nmid \Delta(B / F), \\
\left\{h \in M_{m}\left(B_{v}\right) \mid \bar{h}^{t} h=1\right\}, & \text { otherwise, }\end{cases} \\
U_{x, v} & = \begin{cases}\operatorname{Sp}_{2 m}\left(O_{F_{v}}\right), & \text { if } v \nmid \Delta(B / F), \\
\left\{h \in M_{m}\left(O_{B_{v}}\right) \mid \bar{h}^{t} h=1\right\}, & \text { otherwise, }\end{cases}
\end{aligned}
$$

(iii) $G_{x}\left(\mathbb{Q}_{p}\right)=\prod_{v \mid p} G_{x, v}$, where

$$
G_{x, v}=\left\{\begin{array}{ll}
\operatorname{Sp}_{2 m}\left(F_{v}\right), & \text { if } v \nmid \Delta^{\prime}, \\
\left\{h \in M_{m}\left(B_{v}^{\prime}\right) \mid \bar{h}^{t} h=1\right\}, & \text { otherwise. }
\end{array} .\right.
$$

Take $D=B^{\prime}$ and $V^{\prime}=D^{\oplus m}$ with $\varphi(\underline{x}, \underline{y})=\sum x_{i} \bar{y}_{i}$, and take $L=O_{D}^{\oplus m}$. We compute that

(i)' $G^{\varphi}(\mathbb{R})=\left\{h \in M_{m}(\mathbb{H})^{d} \mid \bar{h}^{t} h=1\right\}$, 
(ii)' for any $\ell$, we have $G_{x}\left(\mathbb{Q}_{\ell}\right)=\prod_{v \mid \ell} G_{v}^{\varphi}$ and $U_{0, \ell}=\prod_{v \mid \ell} U_{0, v}$, where

$$
\begin{aligned}
& G_{v}^{\varphi}= \begin{cases}\operatorname{Sp}_{2 m}\left(F_{v}\right), & \text { if } v \nmid \Delta^{\prime}, \\
\left\{h \in M_{m}\left(B_{v}^{\prime}\right) \mid \bar{h}^{t} h=1\right\}, & \text { otherwise, }\end{cases} \\
& U_{0, v}= \begin{cases}\operatorname{Sp}_{2 m}\left(O_{F_{v}}\right), & \text { if } v \nmid \Delta^{\prime}, \\
\left\{h \in M_{m}\left(O_{B_{v}^{\prime}}\right) \mid \bar{h}^{t} h=1\right\}, & \text { otherwise. }\end{cases}
\end{aligned}
$$

For $\ell \neq p$ and $v \mid \ell$, one has $B_{v}=B_{v}^{\prime}$ and that $v \nmid \Delta(B / F)$ if and only if $v \nmid \Delta^{\prime}$. It follows from computation above that $G_{x, \mathbb{R}} \simeq G_{\mathbb{R}}^{\varphi}$ and $G_{x, \mathbb{Q}_{\ell}} \simeq G_{\mathbb{Q}_{\ell}}^{\varphi}$ for all $\ell$. Since the Hasse principle holds for the adjoint group $G_{x}^{\text {ad }}$, we get $G_{x} \simeq G^{\varphi}$ over $\mathbb{Q}$. We fix an isomorphism and write $G_{x}=G^{\varphi}$. For $\ell \neq p$ and $v \mid \ell$, the subgroups $U_{0, v}$ and $U_{x, v}$ are conjugate, and hence they have the same local volume.

4.2. Applying Theorem 2.2 in our setting (Section 1) and using Lemma 4.2, we get $\operatorname{Mass}\left(\Lambda_{g}^{B}\right)=\operatorname{Mass}\left(G_{x}, U_{x}\right)$. Using the result in Subsection 4.1, we get

$$
\operatorname{Mass}\left(\Lambda_{g}^{B}\right)=\operatorname{Mass}\left(G^{\varphi}, U_{0}\right) \cdot \mu\left(U_{0, p} / U_{x, p}\right),
$$

where $\mu\left(U_{0, p} / U_{x, p}\right)=\left[U_{x, p}: U_{0, p} \cap U_{x, p}\right]^{-1}\left[U_{0, p}: U_{0, p} \cap U_{x, p}\right]$.

\section{LOCAL INDEX $\mu\left(U_{0, p} / U_{x, p}\right)$}

Let $\left(M^{\prime},\langle,\rangle^{\prime}, \iota^{\prime}\right)$ be the covariant Dieudonné module associated to the point $x=$ $\left(A_{0}, \lambda_{0}, \iota_{0}\right)$ in the previous section. Choose an isomorphism $O_{B} \otimes \mathbb{Z}_{p} \simeq M_{2}\left(O_{F} \otimes \mathbb{Z}_{p}\right)$ so that $*$ becomes the transpose. Let $M:=e M^{\prime},\langle\rangle:,=\left.\langle,\rangle^{\prime}\right|_{M}$ and $\iota:=\left.\iota^{\prime}\right|_{O_{F}}$, where $e=\left(\begin{array}{ll}1 & 0 \\ 0 & 0\end{array}\right)$ in $M_{2}\left(O_{F} \otimes \mathbb{Z}_{p}\right)$. The triple $(M,\langle\rangle,, \iota)$ is a superspecial principally quasi-polarized Dieudonné module with compatible $O_{F} \otimes \mathbb{Z}_{p}$-action of rank $g=2 d m$. Let $M=\oplus_{v \mid p} M_{v}$ be the decomposition with respect to the decomposition $O_{F} \otimes \mathbb{Z}_{p}=\oplus_{v \mid p} \mathcal{O}_{v}$; here we write $\mathcal{O}_{v}$ for $O_{F_{v}}$. By the Morita equivalence, we have

$$
U_{x, p}=\operatorname{Aut}_{\mathrm{DM}, O_{B}}\left(M^{\prime},\langle,\rangle^{\prime}\right)=\operatorname{Aut}_{\mathrm{DM}, O_{F}}(M,\langle,\rangle)=\prod_{v \mid p} U_{x, v},
$$

where $U_{x, v}:=\operatorname{Aut}_{\mathrm{DM}, \mathcal{O}_{v}}\left(M_{v},\langle\rangle,\right)$.

Let $W:=W(k)$ be ring of Witt vectors over $k$ and $\sigma$ the absolute Frobenius map on $W$. Let $\mathcal{J}:=\operatorname{Hom}\left(\mathcal{O}_{v}, W\right)$ be the set of embeddings; write $\mathcal{J}=\left\{\sigma_{i}\right\}_{i \in \mathbb{Z} / f_{v} \mathbb{Z}}$ so that $\sigma \sigma_{i}=\sigma_{i+1}$ for all $i$. We identify $\mathbb{Z} / f_{v} \mathbb{Z}$ with $\mathcal{J}$ through $i \mapsto \sigma_{i}$. Decompose $M_{v}=\oplus_{i \in \mathbb{Z} / f_{v} \mathbb{Z}} M_{v}^{i}$ into $\sigma_{i}$-isotypic components $M_{v}^{i}$. One has (1) each component $M_{v}^{i}$ is a free $W$-module of rank $2 m$, which is self-dual with respect to the pairing $\langle\rangle,,(2)\left\langle M_{v}^{i}, M_{v}^{j}\right\rangle=0$ if $i \neq j$, and (3) the operations $F$ and $V$ shift by degree 1 and degree -1 , respectively.

Theorem 5.1. Let $\left(M_{v},\langle\rangle,, \iota\right)$ be as above. There is a symplectic basis $\left\{X_{j}^{i}, Y_{j}^{i}\right\}_{j=1, \ldots, m}$ for $M_{v}^{i}$ such that

(i) $Y_{j}^{i} \in V M_{v}^{i+1}$,

(ii) $F X_{j}^{i}=-Y_{j}^{i+1}$ and $F Y_{j}^{i}=p X_{j}^{i+1}$,

for all $i \in \mathbb{Z} / f_{v} \mathbb{Z}$ and all $j$. 
Proof. We write $f, M$ and $q$ for $f_{v}, M_{v}$ and $q_{v}$, respectively. Suppose that $f=2 c$ is even. Let $N:=\left\{x \in M \mid F^{c} x=(-1)^{c} V^{c} x\right\}$. Since $M$ is superspecial, we have $(*) F^{2} N=p N, \widetilde{N} \otimes_{\mathbb{Z}_{q}} W \simeq M$ and $N=\oplus N^{i}$. Since $\overline{V N^{1}}$ is isotropic with respect to $\langle$,$\rangle in N / p N$, we can choose a symplectic basis $\left\{X_{j}^{0}, Y_{j}^{0}\right\}_{j=1, \ldots, m}$ for $N^{0}$ such that $Y_{j}^{0} \in V N^{1}$ for all $j$. Define $X_{j}^{i}$ and $Y_{j}^{i}$ recursively for $j=1, \cdots, j$ :

$$
X_{j+1}^{i}=p^{-1} F Y_{j}^{i}, \quad Y_{j+1}^{i}=-F X_{j}^{i} .
$$

One has $X_{j+2}^{i}=\frac{-1}{p} F^{2} X_{j}^{i}$ and $Y_{j+2}^{i}=\frac{-1}{p} F^{2} Y_{j}^{i}$; hence

$$
X_{j}^{f}=(-1)^{c} p^{-c} F^{2 c} X_{j}^{0}=X_{j}^{0}, \quad Y_{j}^{f}=(-1)^{c} p^{-c} F^{2 c} Y_{j}^{0}=Y_{j}^{0},
$$

for all $j$. It is easy to see that $\left\{X_{j}^{i}, Y_{j}^{i}\right\}_{j=1, \ldots, m}$ forms a symplectic basis for $N^{i}$.

Suppose that $f=2 c+1$ is odd. Let $N:=\left\{x \in M \mid F^{2 f} x+p^{f} x=0\right\}$. We construct a symplectic basis $\left\{X_{j}^{0}, Y_{j}^{0}\right\}_{j=1, \ldots, m}$ for $N^{0}$ with the properties: $X_{j}^{0} \notin V N^{1}, Y_{j}^{0} \in V N^{1}$ and $Y_{j}^{0}=(-1)^{c+1} p^{-c} F^{f} X_{j}^{0}$ for all $j$. We can choose $X_{1}^{0} \in N^{0} \backslash V N^{1}$ so that $\left\langle X_{1}^{0},(-1)^{c+1} p^{-c} F^{f} X_{1}^{0}\right\rangle \in \mathbb{Z}_{q^{2}}^{\times}$. This follows from the fact that the form $(x, y):=\left\langle x, p^{-c} F^{f} y\right\rangle \bmod p$ is a non-degenerate Hermitian form on $N^{0} / V N^{1}$. Set $Y_{1}^{0}=(-1)^{c+1} p^{-c} F^{f} X_{1}^{0}$ and let $\mu:=\left\langle X_{1}^{0}, Y_{1}^{0}\right\rangle$. From $\left\langle F^{f} X_{1}^{0}, . F^{f} Y_{1}^{0}\right\rangle=\left\langle(-1)^{c+1} p^{c} Y_{1}^{0},(-1)^{c} p^{c+1} X_{1}^{0}\right\rangle$, we get $\mu \in \mathbb{Z}_{q}^{\times}$. Since $\mathbb{Q}_{q^{2}} / \mathbb{Q}_{q}$ is unramified, replacing $X_{1}^{0}$ by a suitable $\lambda X_{1}^{0}$, we get $\left\langle X_{1}^{0}, Y_{1}^{0}\right\rangle=1$. Do the same construction for the complement of the submodule $\left\langle X_{1}^{0}, Y_{1}^{0}\right\rangle$ and use induction; we exhibit such a basis for $N^{0}$.

Define $X_{j}^{i}$ and $Y_{j}^{i}$ recursively for $i=1, \ldots, f$ as (5.2). We verify again that $X_{j}^{f}=X_{j}^{0}$ and $Y_{j}^{f}=Y_{j}^{0}$. It follows from the relation (5.2) that $\left\{X_{j}^{i}, Y_{j}^{i}\right\}_{j=1, \ldots, m}$ forms a symplectic basis for $N^{i}$ for all $i$. This completes the proof.

Proposition 5.2. Notation as above.

(1) If $f_{v}$ is even, then

$$
U_{x, v}=\left\{\left(\begin{array}{cc}
A & B \\
C & D
\end{array}\right) \in \operatorname{Sp}_{2 m}\left(\mathbb{Z}_{q_{v}}\right) \mid B \equiv 0 \bmod p\right\} .
$$

(2) If $f_{v}$ is odd, then

$$
U_{x, v} \simeq\left\{h \in M_{m}\left(O_{B_{v}^{\prime}}\right) \mid \bar{h}^{t} h=1\right\} .
$$

Proof. Let $\phi \in U_{x, v}$. Choose a symplectic basis $\mathcal{B}$ for $M_{v}$ as in Theorem 5.1 Since $\phi$ commutes with the $O_{F}$-action, we have $\phi=\left(\phi_{i}\right)$, where $\phi_{i} \in \operatorname{Aut}\left(M_{v}^{i},\langle\rangle,\right)$. Write $\phi_{i}=\left(\begin{array}{cc}A_{i} & B_{i} \\ C_{i} & D_{i}\end{array}\right) \in \operatorname{Sp}_{2 m}(W)$ using the basis $\mathcal{B}$. Since the map $F$ is injective, $\phi_{0}$ determines the remaining $\phi_{i}$. From $\phi F^{2}=F^{2} \phi$, we have $\phi_{i+2}=\phi_{i}^{(2)}$ (as matrices). Here we write $\phi_{i}^{(n)}$ for $\phi_{i}^{\sigma^{n}}$. From $\phi F=F \phi$ we get $A_{i}^{(1)}=D_{i+1}$, $B_{i}^{(1)}=-p C_{i+1}, p C_{i}^{(1)}=-B_{i+1}$ and $D_{i}^{(1)}=A_{i+1}$.

(1) If $f_{v}$ is even, then $A_{0}, B_{0}, C_{0}, D_{0} \in \mathbb{Z}_{q_{v}}$ and $B_{0} \equiv 0 \bmod p$. This shows (5.3).

(2) Suppose $f_{v}$ is odd. From $\phi_{0}^{\left(f_{v}+1\right)}=\phi_{1}$ we get $A_{0}^{\left(f_{v}\right)}=D_{0}, B_{0}^{\left(f_{v}\right)}=-p C_{0}$, $p C_{0}^{\left(f_{v}\right)}=-B_{0}, D_{0}^{\left(f_{v}\right)}=A_{0}$. Hence

$$
U_{x, v}=\left\{\left(\begin{array}{cc}
A & -p C^{\tau} \\
C & A^{\tau}
\end{array}\right) \in \operatorname{Sp}_{2 m}\left(\mathbb{Z}_{q_{v}^{2}}\right)\right\}
$$


where $\tau$ is the involution of $\mathbb{Q}_{q_{v}^{2}}$ over $\mathbb{Q}_{q_{v}}$. Note that $O_{B_{v}^{\prime}}=\mathbb{Z}_{q_{v}^{2}}[\Pi]$ with $\Pi^{2}=-p$ and $\Pi a=a^{\tau} \Pi$ for all $a \in \mathbb{Z}_{q_{v}^{2}}$. The map $A+C \Pi \mapsto\left(\begin{array}{cc}A & -p C^{\tau} \\ C & A^{\tau}\end{array}\right)$ gives rise to an isomorphism (5.4). This proves the proposition.

Let $\left(V_{0}=\mathbb{F}_{q}^{2 m}, \psi_{0}\right)$ be a standard symplectic space. Let $P$ be the stabilizer of the standard maximal isotropic subspace $\mathbb{F}_{q}<e_{1}, \ldots, e_{m}>$.

Lemma 5.3. $\left|\operatorname{Sp}_{2 m}\left(\mathbb{F}_{q}\right) / P\right|=\prod_{i=1}^{m}\left(q^{i}+1\right)$.

Proof. We have a natural bijection between the group $\operatorname{Sp}_{2 m}\left(\mathbb{F}_{q}\right)$ and the set $\mathcal{B}(m)$ of ordered symplectic bases $\left\{v_{1}, \ldots, v_{2 m}\right\}$ for $V_{0}$. The first vector $v_{1}$ has $q^{2 m}-1$ choices. The first companion vector $v_{m+1}$ has $q^{2 m-1}$ choices as it does not lie in the hyperplane $v_{1}^{\perp}$ and we require $\psi_{0}\left(v_{1}, v_{m+1}\right)=1$. The remaining ordered symplectic basis can be chosen from the complement $\mathbb{F}_{q}<v_{1}, v_{m+1}>^{\perp}$. Therefore, we have proved the recursive formula $\left|\mathrm{Sp}_{2 m}\left(\mathbb{F}_{q}\right)\right|=\left(q^{2 m}-1\right) q^{2 m-1}\left|\mathrm{Sp}_{2 m-2}\left(\mathbb{F}_{q}\right)\right|$. From this, we get

$$
\left|\mathrm{Sp}_{2 m}\left(\mathbb{F}_{q}\right)\right|=q^{m^{2}} \prod_{i=1}^{m}\left(q^{2 i}-1\right) .
$$

We have

This yields

$$
P=\left\{\left(\begin{array}{cc}
A & B \\
0 & D
\end{array}\right) ; A D^{t}=I_{m}, B A^{t}=A B^{t}\right\}
$$

$$
|P|=q^{\frac{m^{2}+m}{2}}\left|\mathrm{GL}_{m}\left(\mathbb{F}_{q}\right)\right|=q^{m^{2}} \prod_{i=1}^{m}\left(q^{i}-1\right) .
$$

From (5.5) and (5.6), we prove the lemma.

By Proposition 5.2 and Lemma 5.3 we get

Theorem 5.4. One has

$$
\mu\left(U_{0, p} / U_{x, p}\right)=\prod_{v \mid p} \mu\left(U_{0, v} / U_{x, v}\right)=\prod_{v \mid p, v \nmid \Delta^{\prime}} \prod_{i=1}^{m}\left(q_{v}^{i}+1\right) .
$$

Plugging the formula (5.7) in the formula (4.4), we get the formula (1.3). The proof of Theorem 1.2 is complete.

Acknowledgments. The present work relies on Shimura's paper [7] and is also inspired by W.-T. Gan and J.-K. Yu's paper 3]. It is a great pleasure to thank them.

\section{REFERENCES}

[1] T. Ekedahl, On supersingular curves and supersingular abelian varieties. Math. Scand. 60 (1987), 151-178.

[2] W. T. Gan, J. P. Hanke, and J.-K. Yu, On an exact mass formula of Shimura. Duke Math. J. 107 (2001), 103-133.

[3] W. T. Gan and J.-K. Yu, Group schemes and local densities. Duke Math. J. 105 (2000), 497-524.

[4] K. Hashimoto and T. Ibukiyama, On class numbers of positive definite binary quaternion hermitian forms, J. Fac. Sci. Univ. Tokyo 27 (1980), 549-601. 
[5] G. Prasad, Volumes of $S$-arithmetic quotients of semi-simple groups. Inst. Hautes Études Sci. Publ. Math. 69 (1989), 91-117.

[6] M. Rapoport and Th. Zink, Period Spaces for p-divisible groups. Ann. Math. Studies 141, Princeton Univ. Press, 1996.

[7] G. Shimura, Some exact formulas for quaternion unitary groups. J. Reine Angew. Math. 509 (1999), 67-102.

[8] C.-F. Yu, Simple mass formulas on Shimura varieties of PEL-type. math.NT/0603451, 15 pp.

Institute of Mathematics, Academia Sinica, 128 Academia Rd. Sec. 2, Nankang, Taipei, TAiwan, AND NCTS (TAIPEi Office)

E-mail address: chiafu@math.sinica.edu.tw

MaX-Planck-Institut für Mathematik, Vivatsgasse 7, Bonn, 53111, Germany 